\title{
Pseudogap metal induced by long-range Coulomb interactions
}

\author{
K. Driscoll $\odot$, A. Ralko $\odot$, and S. Fratini $\oplus^{*}$ \\ Institut Néel, Centre National de la Recherche Scientifique and Université Grenoble Alpes, 38042 Grenoble, France
}

(Received 6 October 2020; accepted 25 April 2021; published 7 May 2021)

\begin{abstract}
In correlated electron systems the metallic character of a material can be strongly suppressed near an integer concentration of conduction electrons as Coulomb interactions forbid the double occupancy of local atomic orbitals. While the Mott-Hubbard physics arising from such on-site interactions has been largely studied, several unexplained phenomena observed in correlated materials challenge this description and call for the development of new ideas. Here we explore a general route for obtaining correlated behavior that is decidedly different from the spin-related Mott-Hubbard mechanism and instead relies on the presence of unscreened, long-range Coulomb interactions. We find a pseudogap metal phase characterized by a divergent quasiparticle mass and the opening of a Coulomb pseudogap in the electronic spectrum. The destruction of the Fermi-liquid state occurs because the electrons move in a nearly frozen, disordered charge background, as collective charge rearrangements are drastically slowed down by the frustrating nature of long-range potentials on discrete lattices. The present pseudogap metal realizes an early conjecture by Efros, that a soft Coulomb gap should appear for quantum lattice electrons with strong unscreened interactions due to self-generated randomness.
\end{abstract}

DOI: 10.1103/PhysRevB.103.L201106

\section{INTRODUCTION}

The Mott metal-insulator transition (MIT) is one of the cornerstones of modern condensed matter physics [1,2]. Originally devised to explain the cause of the insulating state in narrow-band materials with partially filled bands, modern focus has shifted to understanding the anomalous properties of metals that arise near the MIT and their possible consequences in stabilizing other phases, including superconductivity. Most theoretical developments in the field have relied on the Hubbard model and its variants, where the Coulomb repulsion between electrons is reduced to its strongest (on-site) termneglecting all nonlocal terms from the outset. Based on these models and the techniques that have been developed and applied to the problem, we now have a broad understanding of the physics of strongly correlated electron systems. Despite this widespread success several experimental puzzles in quantum materials remain however unexplained [3-7], which drives us to revisit the implicit assumptions in the MottHubbard description. To this aim we solve a lattice model which explicitly includes long-range electron-electron interactions, demonstrating that these can give rise to strongly correlated behavior physically distinct from the Mott type. Unrelated to the spin degrees of freedom, we find strong mass renormalization caused by the buildup of nonlocal charge correlations dressing the quasiparticles, which is accompanied by the opening of a pseudogap in the single-particle spectrum. This happens at the approach of Wigner crystallization, where the fluctuating charge density is collectively slowed down and behaves effectively as a nearly frozen random medium,

\footnotetext{
*simone.fratini@neel.cnrs.fr
}

thereby enabling the Efros-Shklovskii (ES) Coulomb gap phenomenon.

\section{MODEL AND METHODS}

We study spinless electrons interacting through a longrange repulsive potential $V(R)=V \times(R / a)^{-\alpha}$ on a twodimensional lattice, as described by the following Hamiltonian [8]:

$$
H=-t \sum_{\langle i j\rangle} c_{i}^{\dagger} c_{j}+\frac{1}{2} \sum_{i j} V\left(R_{i j}\right)\left(\hat{n}_{i}-n\right)\left(\hat{n}_{j}-n\right) .
$$

Here $c_{i}^{\dagger}$ and $c_{i}$ are creation and annihilation operators for electrons on local atomic orbitals; $\hat{n}_{i}$ is the local density operator; $t$ is the hopping matrix element between nearest-neighbor sites, which we take to be isotropic; and $n=1 / 2$ is the average electron concentration. The strength of the interactions is controlled by $V$, the value of the potential at one lattice spacing $a$ (which we set as the unit length). For illustrative purposes we choose to present results for the triangular lattice, but our findings are not specific to this particular lattice geometry (see Supplemental Material [9]). We explore the full phase diagram of the model, taking the power-law exponent $\alpha$ as a continuous parameter. The chosen form of $V(R)$ includes the pristine Coulomb potential $V(R) \sim 1 / R(\alpha=1)$ and the commonly studied nearest-neighbor repulsion characteristic of the extended Hubbard model $(\alpha=\infty)$, as well as the dipolar form $V(R) \sim 1 / R^{3}$ of the two-dimensional electron gas near a metallic gate $(\alpha=3)$.

Equation (1) is solved numerically via both Lanczos and brute force exact diagonalization at zero temperature on finitesize clusters with $N_{s}=12,18,24$ sites [10]. Finite-size errors on the kinetic part are minimized by averaging over twisted 


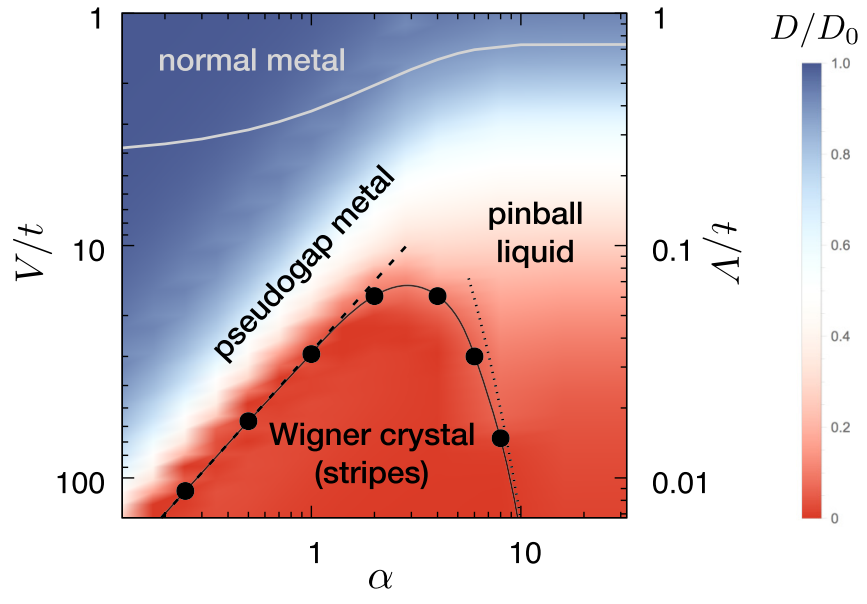

FIG. 1. Phase diagram of the triangular lattice model with longrange interactions. The line and the full symbols indicate the metal-insulator transition to a stripe-ordered Wigner crystal, signaled by the vanishing of the Drude weight $D\left(N_{s}=18\right.$ sites, using 121 TBCs). The color map is the Drude weight (697 grid points, $N_{s}=$ 12 sites, 400 TBCs). The gray dashed line is the charge ordering transition as obtained from the random-phase approximation, which ignores correlations and does not capture the extreme fragility of the Wigner crystal. The black dashed and dotted lines are strongcoupling estimates for the Wigner crystal melting (see text).

boundary conditions (TBCs) both at fixed particle number [11] and in the grand-canonical ensemble [12,13], which restores the exact $N_{s} \rightarrow \infty$ result in the noninteracting limit, $V / t \rightarrow 0$ (see Supplemental Material [9]). For the interaction part, we extend the cluster size to the thermodynamic limit by considering infinitely repeated simulation cells. We perform the corresponding lattice sums using the Ewald summation method [8], which ensures that the electrostatic (Madelung) energy of periodic configurations is exactly recovered in the classical limit, $t / V \rightarrow 0$. Details on the calculation of observables can be found in the Supplemental Material [9].

\section{PHASE DIAGRAM}

Figure 1 presents the phase diagram of the model as a function of the power-law exponent $\alpha$. Four different regions are found: Normal metal at weak interaction strengths, and the pinball liquid (PL), stripe-ordered Wigner crystal, and pseudogap metal at strong interactions. The origin of all three strongly interacting phases can be understood starting from the classical limit of the model. At $t / V=0$, for nearestneighbor repulsive potentials ( $\alpha \rightarrow \infty$, right side of Fig. 1) there exist infinitely many classical configurations, where part of the particles ("pins") are located on a superlattice with threefold periodicity, the other particles ("balls") being randomly distributed on the remaining honeycomb lattice [14], all having the same Madelung energy $E_{\mathrm{Mad}} / N_{s}=V / 2$. This degeneracy is lifted by quantum fluctuations: as soon as $t / V>0(V / t<\infty)$, minimizing the kinetic term for the balls provides a net energy gain $\propto t$, identifying a unique macroscopic ground state- the PL [14]. This state has strong threefold correlations reminiscent of the classical limit and a weakly metallic character, which progressively evolves into a normal metal upon reduction of the interaction strength $V / t[15]$.

Long-range interactions also immediately lift the massive degeneracy characterizing the classical limit [16]. As soon as $\alpha<\infty$ (bottom part of Fig. 1), the interactions beyond nearest neighbors favor linear stripe configurations, which become the most stable states at $t=0$. The stripe phase, which is the lattice analog of a Wigner crystal [17], remains insulating in the presence of quantum fluctuations at small $t>0$, as indicated by the vanishing of the Drude weight [Figs. 1 and 2(c)]. The pinball liquid can still be stabilized above some critical value of $t / V$ as long as $\alpha \gtrsim 2$. The potential-energy difference with the classically more stable stripe configurations behaves asymptotically as $\Delta E \propto V /\left(R_{2}\right)^{\alpha}$, with $R_{2}=$ $\sqrt{3}$ the second neighbor distance. The transition from stripes to PL occurs when the kinetic-energy gain associated with the itinerant carriers overcomes such energy difference, leading to $(V / t)_{c} \propto 3^{\alpha / 2}$ (dotted line in Fig. 1).

\section{SUPPRESSION OF ORDER BY THE LONG-RANGE INTERACTIONS}

Reducing the long-range exponent below $\alpha \leqslant 2$ reveals a domelike shape, with the stripe-ordered insulator becoming more and more unstable with increasing range of interactions. The fragility of Wigner crystal order is a known feature of long-range interactions in the continuum: In the jellium model with pure Coulomb repulsion $(\alpha=1)$, the ordered state melts due to the existence of extremely soft, shear collective modes that are easily accessible via a low energetic cost $[8,18,19]$. For this reason, the ratio of interaction to kinetic energy, as given by the appropriate dimensionless interaction parameter, is large at the transition: $r_{s} \simeq 31$ for quantum electrons in $d=$ 2 [20]. Strong interaction effects then naturally persist into the metallic state beyond melting, causing short-range spatial correlations that are reminiscent of those in the ordered phase [21], and a consequently large correlation energy.

Analogously, for quantum lattice electrons as considered here, long-range interactions favor charge fluctuations, destabilizing the Wigner crystal (stripe) order and uncovering the correlated metallic state that lies underneath. To assess this effect, we again resort to the $t / V \rightarrow 0$ limit and evaluate the energy required to create a defect of the ordered pattern, $E_{d}$ [17], which is obtained by displacing a carrier from its equilibrium position on the stripe to a neighboring unoccupied site on the lattice. While this energy cost is exactly $E_{d}=V$ in the nearest-neighbor limit $(\alpha \rightarrow \infty)$, it is steadily suppressed upon increasing the range of the interactions. As a result, defects are more and more easily created by quantum fluctuations when $t>0$. The quantum melting transition occurs through proliferation of such defects when $t \sim E_{d}[17,22,23]$. From the asymptotic expression $E_{d} \simeq 0.469 \mathrm{~V} \alpha$ we obtain $(V / t)_{c} \propto 1 / \alpha$ for small $\alpha$, as observed in Fig. 1 (black dashed line). For comparison we show the transition predicted by the random-phase approximation (gray dashed line) [24]. This approximation captures the onset of local charge order but it completely misses the fragility of long-range order at small $\alpha$, which arises from correlations beyond mean-field level. Remarkably, the pure Coulomb case $\left[\alpha=1,(V / t)_{c} \simeq 29\right.$, corresponding to $\left.r_{s}=7.2\right]$ lies well on the asymptotic "small $\alpha$ " 

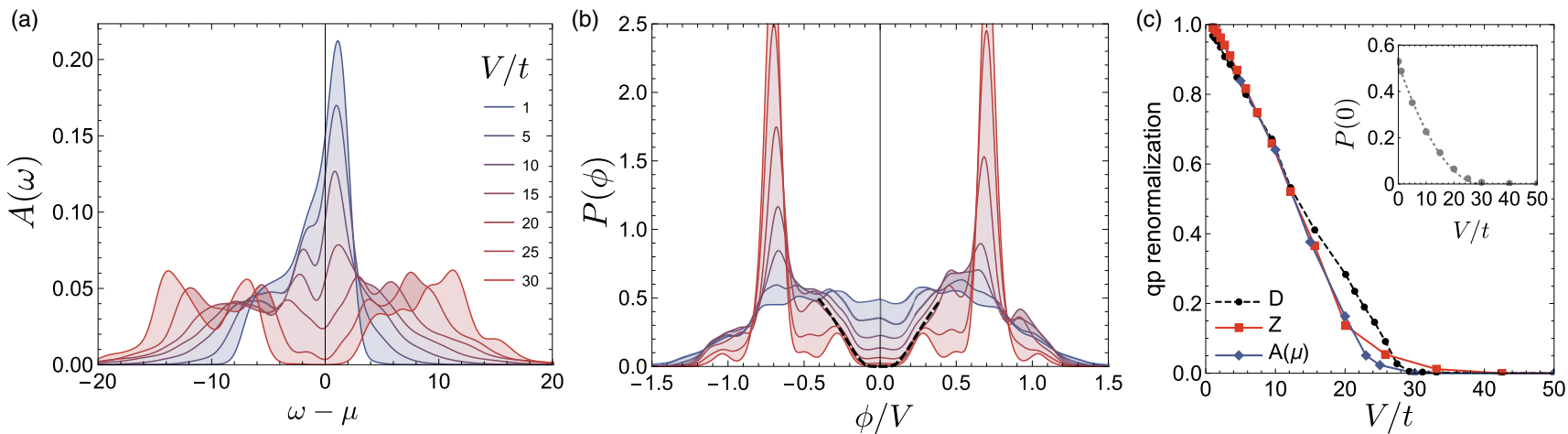

FIG. 2. Pseudogap metal. (a) Spectral function $A(\omega)$ at $\alpha=1$ and $N_{s}=18$ and averaged over 16 TBCs, illustrating the pseudogap phenomenon. All spectra are smoothened by a Gaussian broadening $\delta=0.7 t$. (b) Distribution of classical local potentials $P(\phi)$ (broadening $\delta=0.05 V$ ), showing the opening of the Coulomb gap; the dashed line is $P \sim e^{-V / \xi|\phi|}$ with $\xi=2$; the values of $V / t$ and the color code are the same as in (a). (c) Drude weight $D$ (averaged over $121 \mathrm{TBCs}$ ) normalized by the exact noninteracting value $D_{0}=0.247$, quasiparticle weight $Z$ (angular averaged over the Fermi surface), and density of states $A(\mu)$ at the Fermi energy, divided by the noninteracting value. The inset shows $P(0)$, tracking the plasma dip. The dashed line is $P(0) \sim\left|V-V_{c}\right|^{2}$.

side of the dome: in this regime we have $E_{d} \ll V$, signifying that the local, short-range energy scale $V$ and the global, longrange scale responsible for collective behavior and melting are indeed well separated. As we shall see, this separation of energy scales has profound consequences on the electronic properties of the metal.

\section{PSEUDOGAP METAL}

Figure 2(a) illustrates the evolution as a function of $V / t$ of the local single-particle spectral function $A(\omega)$ in the metallic state. As the interaction strength increases, a pseudogap opens at the Fermi energy $(\omega=\mu)$, which progressively deepens and broadens as excitations move towards high energies, $\omega \sim$ $V$. The density of states (DOS) at the Fermi energy, $A(\mu)$, falls approximately linearly with $V / t$, then flattens deep in the pseudogap phase and eventually vanishes at the MIT at $(V / t)_{c} \simeq 29$ [Fig. 2(c)]; the pseudogap coalesces into a hard gap in the stripe phase beyond this value. Figure S17 in Supplemental Material [9] shows analogous results obtained on the square lattice, demonstrating that the source of frustration responsible for the pseudogap formation originates from the long-range interactions, and not the lattice geometry [25].

Concomitant with the development of the pseudogap in the one-particle spectrum, electronic correlations build up, signalled by a steady decrease of both the Drude weight, $D$, and the quasiparticle weight, $Z$, with the latter following closely the behavior of $A(\mu)$ [Fig. 2(c)]. At the MIT both $D$ and $Z$ vanish, indicating the divergence of the quasiparticle mass, $m^{*} / m_{b} \propto 1 / Z$, and of the optical effective mass, $m_{\mathrm{opt}}^{*} / m_{b}=D_{0} / D$ (see Supplemental Material [9]). Strikingly, the mechanism for mass divergence at work here is radically different from the spin-related mechanism involved in the bandwidth-controlled Mott-Hubbard transition. In the case of the Mott-Hubbard MIT, the spectral function features a quasiparticle peak that remains pinned at the Fermi energy, and the shrinking of which with $Z$ mostly causes the divergence of the effective mass [1,2]. Here no peak narrowing is found, and it is instead the value of the renormalized DOS at the Fermi energy, $A(\mu)$, that falls continuously to zero controlling the quasiparticle renormalization [Fig. 2(c)].

\section{SELF-GENERATED RANDOMNESS AND SHORT-RANGE CORRELATIONS}

The pseudogap phenomenon revealed in the preceding paragraphs is strongly reminiscent of the soft Coulomb gap characteristic of disordered insulators. There, stability arguments imply that the DOS of an interacting electron system in the presence of quenched disorder must vanish at the Fermi energy [26], due to their long-range mutual interactions. Similar physics was also reported in clean classical Coulomb liquids, where it was shown that the long-distance potentials from electrons beyond the correlation length, when taken collectively, act as a source of (self-generated) randomness [8,16,27-29]. The observations presented in Fig. 2 highlight that the phenomenon of self-generated randomness and the associated Coulomb pseudogap exist also in the clean quantum case, as hypothesized by Efros almost three decades ago [27]. The resemblance between the quantum phase diagram Fig. 1 and its classical analog determined in Ref. [8] is striking.

To track the origin of the pseudogap, we determine the distribution of electrostatic site energies in the quantum ground state $|\psi\rangle$, which can be evaluated as $P\left(\phi_{i}\right)=\langle\psi| \delta\left[\phi_{i}-\right.$ $\left.\sum_{j \neq i} V\left(R_{i j}\right)\left(\hat{n}_{j}-n\right)\right]|\psi\rangle$ (the site index can be ignored as this quantity is translationally invariant in the present case). For classical electrons, $P(\phi)$ would reduce to the density of states studied in the Efros-Shklovskii soft gap problem [26]. In the quantum case, it represents the fluctuating background where the electron motion takes place.

Figure 2(b) shows that, prior to the pseudogap opening observed in the full electronic spectrum, a broad dip develops already in the distribution of site potentials. Interestingly, its shape at the transition is compatible with that caused by shortrange charge correlations in self-generated Coulomb glasses, $P \sim e^{-V / \xi|\phi|}$ (dashed line) [29]. There, the correlation hole that forms around electrons in order to minimize their mutual interactions was shown to deplete the classical DOS below the ES bound, $P_{\mathrm{ES}} \sim|\phi|\left(P_{\mathrm{ES}} \sim|\phi|^{d / \alpha-1}\right.$ in the general case 
in $d$ dimensions and exponent $\alpha$ ). Such correlation hole, or "electronic polaron," is a common feature of electron liquids with unscreened Coulomb interactions [21]. Its buildup within the pseudogap phase is confirmed here by direct evaluation of the charge correlation function (see Supplemental Material [9]). We have verified that our method fully recovers the prediction $P_{\mathrm{ES}} \sim|\phi|$ upon suppressing short-range correlations via the introduction of extrinsic (quenched) disorder, and that the pseudogap disappears for short-range interactions $\alpha>D$, demonstrating that the observed pseudogap is the consequence of the long-range Coulomb interaction.

\section{SOFT COLLECTIVE EXCITATIONS}

The results presented above demonstrate strongly correlated behavior arising from long-range charge-charge interactions, which is unrelated to the paradigmatic Mott mechanism. Our findings indicate that in systems with unscreened, longrange interactions, the collective charge fluctuations are able to provide a self-generated random environment, thereby enabling precursors of the Efros-Shklovskii Coulomb gap phenomenon. This fluctuating environment is polarizable and responds to the motion of the individual electrons, being ultimately responsible for the mass enhancement via the formation of electronic polarons.

The existence of a Coulomb pseudogap necessarily implies that there is a marked separation of timescales between the (fast) motion of individual electrons and the (much slower) global rearrangements of the charge at long distances: The idea being that the charge fluctuation background is almost frozen, being collectively jammed by the mutual interactions among its constituents $[8,18,19,28,30]$. We can actually provide quantitative support to this statement, by evaluating the spectrum of charge fluctuations, $\mathcal{D}(\omega)=$ $\sum_{m}\left|\left\langle m\left|\hat{\rho}_{q}\right| \psi\right\rangle\right|^{2} \delta\left[\omega-\left(E_{m}-E_{0}\right)\right]$, where $\hat{\rho}_{q}$ is the Fourier transform of the charge density $\hat{n}_{i}$, and $m$ and $E_{m}$ are all the eigenstates and eigenenergies. Figure 3(a) shows that there is a strong contribution to the spectrum that is soft throughout the pseudogap phase, peaking at $\omega_{\text {coll }} \simeq 0.2-0.25 t$ [see also Fig. 3(b)]. This is about 30-50 times lower than the free-electron bandwidth, $9 t$. This collective contribution is mostly unrelated to the critical mode responsible for stripe ordering [wave vector $q \equiv M$, which instead softens only at the MIT, see inset and Fig. 3(b)]. It arises instead from a diffuse region near the edges of the Brillouin zone [16], indicative of the existence of many competing orders being frustrated by the long-range interactions $[16,28]$. Translated to real space, these zone-boundary features correspond to the local (short-distance) dipoles postulated in Ref. [30], arising from frustrated charge correlations. The fact that within the pseudogap phase the metallic character measured by the Drude weight, $D / D_{0}$, is larger than that implied by the oneparticle residue $Z$ alone, as observed in Fig. 2(c), suggests that such collective modes could be actively contributing to charge transport as an additional conduction channel $[17,31]$.

\section{CONCLUDING REMARKS}

The existence of self-generated randomness with a suppressed energy scale implies an equally suppressed tem-
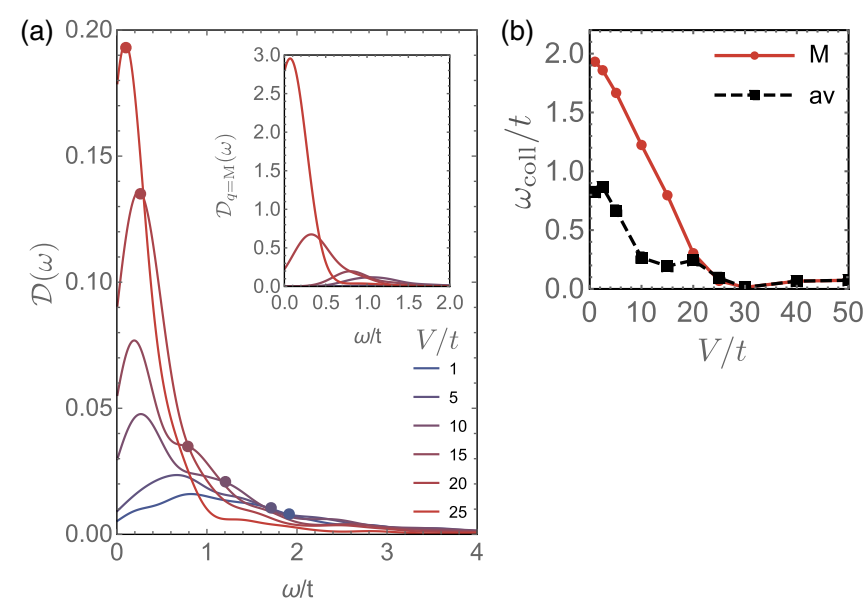

FIG. 3. Soft collective excitations in the pseudogap phase. (a) Spectral function $\mathcal{D}(\omega)$ of the charge fluctuations, averaged over the Brillouin zone (Gaussian broadening $\delta=0.2 t$ ). The inset shows the same quantity evaluated at the stripe-ordering wave vector $q=$ $M$; this critical mode is also visible as a shoulder in the main panel (circles). (b) Frequency of the boson peak, controlling the timescale of the charge fluctuations.

perature scale at which quantum coherence is lost. The collapse onto classical behavior should be further enhanced by the fact that the random potentials possess a continuous spectrum [Fig. 2(b)], thus providing a natural source of electron decoherence [32]. This could explain, for example, the puzzling behavior observed in the quarter-filled organic compounds $\theta$-(BEDT-TTF $)_{2} \mathrm{X}$. In these materials, the electron liquid shows precursors of glassiness despite the absence of structural disorder [33,34], that are surprisingly well captured by classical models [16]. Moreover, in agreement with the results found here, these materials display frustrated metastable orders (seen as diffuse spots in x-ray-diffraction images) that compete with the stripes [33]. Above an extremely low Fermi temperature, $T_{\mathrm{FL}}^{*} \sim 20 \mathrm{~K}$, which is two orders of magnitude lower than predicted by band-structure arguments, the resistivity displays strange metal behavior with an approximately linear temperature dependence [35,36] compatible with strong scattering by low-energy bosonic modes. The system also features a displaced Drude peak in the optical conductivity, suggestive of disorder-induced localization, indicating that self-generated randomness could also be playing a key role in the charge transport mechanism $[37,38]$.

Due to the general nature of the effects revealed here, it will be interesting to investigate their relevance in other quantum materials exhibiting bad metallic behavior $[3,6]$, including those near integer fillings where long-range interactions are customarily neglected. In these systems, the reduced screening ability of electrons at the onset of the Mott transition should imply that long-range potentials play a significant role $[7,28,30]$, therefore contributing to their anomalous thermodynamic and transport properties: the importance of long-range interactions and the ensuing nearly classical behavior of charge fluctuations could bring the $T$-linear behavior of the resistivity, which characterizes 
correlated electrons at very high temperatures [39], down to the experimentally relevant temperature range. Generally speaking, the interplay of Wigner and Mott physics should provide a promising direction in research on strongly correlated materials.

\section{ACKNOWLEDGMENTS}

We thank S. Ciuchi, L. de' Medici, and V. Dobrosavljević for enlightening discussions. K.D. acknowledges the European Union's Horizon 2020 Research and Innovation program under Marie Skłodowska-Curie Grant No. 754303.
[1] A. Georges, G. Kotliar, W. Krauth, and M. J. Rozenberg, Rev. Mod. Phys. 68, 13 (1996).

[2] M. Imada, A. Fujimori, and Y. Tokura, Rev. Mod. Phys. 70, 1039 (1998).

[3] J. A. N. Bruin, H. Sakai, R. S. Perry, and A. P. Mackenzie, Science 339, 804 (2013).

[4] B. Keimer, S. A. Kivelson, and M. R. Norman, S. Uchida, and J. Zaanen, Nature (London) 518, 179 (2015).

[5] B.-X. Zheng, C.-M. Chung, P. Corboz, G. Ehlers, M.-P. Qin, R. M. Noack, H. Shi, S. R. White, S. Zhang, and G. K.-L. Chan Science 358, 1155 (2017).

[6] L. V. Delacrétaz, B. Goutéraux, S. A. Hartnoll, and A. Karlsson, SciPost Phys. 3, 025 (2017).

[7] B. Padhi, C. Setty, and P. W. Phillips, Nano Lett. 18, 6175 (2018).

[8] Y. Pramudya, H. Terletska, S. Pankov, E. Manousakis, and V. Dobrosavljević, Phys. Rev. B 84, 125120 (2011).

[9] See Supplemental Material at http://link.aps.org/supplemental/ 10.1103/PhysRevB.103.L201106 for full calculation details and additional results.

[10] M. Mambrini, Ph.D. thesis, Université Paul Sabatier, Toulouse, France, 2002.

[11] D. Poilblanc, Phys. Rev. B 44, 9562 (1991).

[12] C. Gros, Z. Phys. B 86, 359 (1992).

[13] T. Koretsune, Y. Motome, and A. Furusaki, J. Phys. Soc. Jpn. 76, 074719 (2007).

[14] C. Hotta and N. Furukawa, Phys. Rev. B 74, 193107 (2006).

[15] M. Miyazaki, C. Hotta, S. Miyahara, K. Matsuda, and N. Furukawa, J. Phys. Soc. Jpn. 78, 014707 (2009).

[16] S. Mahmoudian, L. Rademaker, A. Ralko, S. Fratini, and V. Dobrosavljević, Phys. Rev. Lett. 115, 025701 (2015).

[17] S. Fratini and J. Merino, Phys. Rev. B 80, 165110 (2009).

[18] B. Spivak, Phys. Rev. B 64, 085317 (2001).
[19] A. F. Andreev and Yu. A. Kosevich, Zh. Eksp. Teor. Fiz. 77, 2518 (1979) [Sov. Phys. JETP 50, 1218 (1979)].

[20] N. D. Drummond and R. J. Needs, Phys. Rev. Lett. 102, 126402 (2009).

[21] G. D. Mahan, Many-Particle Physics, 3rd ed. (Plenum, New York, 2000).

[22] A. F. Andreev and I. M. Lifshitz, Zh. Eksp. Teor. Fiz. 56, 2057 (1969) [Sov. Phys. JETP 29, 1107 (1969)].

[23] E. V. Tsiper and A. L. Efros, Phys. Rev. B 57, 6949 (1998).

[24] L. Cano-Cortés, A. Ralko, C. Février, J. Merino, and S. Fratini, Phys. Rev. B 84, 155115 (2011).

[25] L. Rademaker, Y. Pramudya, J. Zaanen, and V. Dobrosavljević, Phys. Rev. E 88, 032121 (2013).

[26] A. L. Efros and B. I. Shklovskii, J. Phys. C 8, L49 (1975).

[27] A. L. Efros, Phys. Rev. Lett. 68, 2208 (1992).

[28] J. Schmalian and P. G. Wolynes, Phys. Rev. Lett. 85, 836 (2000).

[29] L. Rademaker, Z. Nussinov, L. Balents, and V. Dobrosavljević, New J. Phys. 20, 043026 (2018).

[30] V. J. Emery and S. A. Kivelson, Phys. C 209, 597 (1993).

[31] S. Caprara, C. Di Castro, S. Fratini, and M. Grilli, Phys. Rev. Lett. 88, 147001 (2002).

[32] A. O. Caldeira and A. J. Leggett, Phys. Rev. Lett. 46, 211 (1981).

[33] F. Kagawa et al., Nat. Phys. 9, 419 (2013).

[34] F. Nad, P. Monceau, and H. M. Yamamoto, Phys. Rev. B 76, 205101 (2007).

[35] K. Takenaka, M. Tamura, N. Tajima, H. Takagi, J. Nohara, and S. Sugai, Phys. Rev. Lett. 95, 227801 (2005).

[36] T. Sato et al., Nat. Mater. 18, 229 (2019).

[37] M. Kaveh and N. F. Mott, J. Phys. C: Solid State Phys. 15, L707 (1982).

[38] S. Fratini, D. Mayou, and S. Ciuchi, Adv. Funct. Mater. 26, 2292 (2016).

[39] C. H. Mousatov, I. Esterlis, and S. A. Hartnoll, Phys. Rev. Lett. 122, 186601 (2019). 